\title{
May home confinement during the COVID-19 outbreak worsen the global burden of myopia?
}

\author{
Marco Pellegrini ${ }^{1} \cdot$ Federico Bernabei ${ }^{1} \cdot$ Vincenzo Scorcia $^{2} \cdot$ Giuseppe Giannaccare $^{2}$ (D
}

Received: 19 April 2020 / Revised: 19 April 2020 / Accepted: 26 April 2020 / Published online: 4 May 2020

(C) Springer-Verlag GmbH Germany, part of Springer Nature 2020

Keywords COVID-19 · Coronavirus · Myopia - Quarantine myopia · Dear Editor,

The novel coronavirus (2019-nCoV) that originated in Wuhan (China) has quickly spread all over the world. As of April 12, 2020, there have been with more than 1,600,000 confirmed cases and 100,000 deaths globally [1]. To restrict contact among individuals, governments have imposed unprecedented public health measures such as travel bans, workplace distancing, and schools closure. The aim of these measures is to reduce the size of the outbreak as well as its peak in order to decrease the amount of stress on healthcare systems.

In a recent correspondence on Lancet, Wang and colleagues pointed out that the measures ordered by the Chinese government to limit the spread of the novel coronavirus, and in particular schools closure, might have negative effects on children's physical and mental health [2]. In fact, the authors stated that when children are out of school, they are physically less active and have longer screen time, irregular sleep pattern, and less favorable diets resulting in weight gain and loss of cardiorespiratory fitness. In addition, the psychological sphere can also be affected by this forced change of lifestyle.

We would like to add another piece to the puzzle of potential impacts of home confinement on children, which is the increased risk of myopia (namely, "quarantine myopia"). The prevalence of myopia has rapidly increased over the past few decades, particularly in countries from East and Southeast Asia. Insufficient time spent outdoors is recognized as an important risk factor for its development [3]. Furthermore, the duration and intensity of near work activities (e.g., reading and

Giuseppe Giannaccare

giuseppe.giannaccare@gmail.com

1 Ophthalmology Unit, S.Orsola-Malpighi University Hospital, Bologna, Italy

2 Department of Ophthalmology, University Magna Græcia of Catanzaro, Viale Europa, 88100, Germaneto, Catanzaro, Italy writing) are also associated with myopia [4]. The mechanisms of visual feedback regulating the eye growth are complex and not completely understood. Factors that may mediate the relationship between time spent outdoors and myopia include the brightness and chromatic spectrum of light, the energy at high spatial frequencies, the peripheral defocus, and the circadian rhythms [3].

According to UNESCO, more than 160 countries have closed schools in the attempt at containing the spread of COVID-19, and this measure involves over $87 \%$ of world's student population [5]. An important consequence of home confinement on children's health could be the development and/or the worsening of myopia. Since the exact duration of exposure is currently undetermined, it is difficult to estimate the effect from a refractive standpoint. A recent modeling study on influenza reported that in case of severe pandemics, the reduction of the cumulative disease incidence could be possible only with school dismissal lasting 16 weeks or longer [6]. A prolonged home confinement would very likely have a significant impact on the global incidence of myopia. This represents a serious public health concern for two main reasons. First, uncorrected myopia represents a major cause of visual disability in children, particularly in low- and middleincome countries. Second, high myopia in the current pediatric population is linked to a high risk of potentially sightthreatening complications in the next generations of elderly.

A range of new interventions for reducing the progression of myopia in children have now reached high level of evidence [7]. A recent network analysis indicated that the most effective options are muscarinic antagonists such as atropine and pirenzepine, whereas certain specially designed contact lenses including orthokeratology and peripheral defocus modifying contact lenses have moderate effects [7]. Therefore, a post-pandemic ophthalmological surveillance program for children with myopia involving decision-making based on demographical and clinical characteristics, risk factors, and 
individual preference should be considered in order to better control disease progression.

\section{Compliance with ethical standards}

Conflict of interest The authors declare that they have no conflict of interest.

\section{References}

1. WHO. (2020) Coronavirus disease 2019 (COVID-19) situation report-83. April 12. https://www.who.int/docs/default-source/ coronaviruse/situation-reports/20200412-sitrep-83-covid-19.pdf? sfvrsn=697ce98d_4. Accessed 18 April 2020

2. Wang G, Zhang Y, Zhao J, Zhang J, Jiang F (2020) Mitigate the effects of home confinement on children during the COVID-19 outbreak. Lancet. Accessed 4 March 2020. https://doi.org/10.1016/ S0140-6736(20)30547-X
3. Lingham G, Mackey DA, Lucas R, Yazar S (2019) How does spending time outdoors protect against myopia? A review. Br J Ophthalmol. Accessed 13 November 2019. https://doi.org/10.1136/ bjophthalmol-2019-314675

4. Wen L, Cao Y, Cheng Q et al (2020) Objectively measured near work, outdoor exposure and myopia in children. Br J Ophthalmol Accessed 19 February 2020. https://doi.org/10.1136/bjophthalmol2019-315258

5. UNESCO. COVID-19 Educational Disruption and Response. https://en.unesco.org/themes/education-emergencies/coronavirusschool-closures. Accessed 18 April 2020

6. Germann TC, Gao H, Gambhir M et al (2019) School dismissal as a pandemic influenza response: when, where and for how long? Epidemics 28:100348. https://doi.org/10.1016/j.epidem.2019. 100348

7. Huang J, Wen D, Wang Q et al (2016) Efficacy comparison of 16 interventions for myopia control in children: a network meta-analysis. Ophthalmology 123:697-708. https://doi.org/10.1016/j.ophtha. 2015.11.010

Publisher's note Springer Nature remains neutral with regard to jurisdictional claims in published maps and institutional affiliations. 